\title{
Evolutionary algorithm for de novo molecular design with multi-dimensional constraints
}

\author{
Robert H. Herring III and Mario R. Eden ${ }^{*}$ \\ Department of Chemical Engineering, Auburn University, Auburn 36849, USA
}

\begin{abstract}
An evolutionary approach for solving molecular design problems with descriptors of varying dimensionality has been developed. Spatial fragment based descriptors are employed to generate candidate solutions within a population, which is evolved through the application of genetic operators towards an improved fitness. The candidate molecules are represented as graphs, and as such, customized operators of crossover and mutation have been developed to be compatible with this representation. The search space is conveniently represented through limitations on the occurrence of each fragment, as defined by the chosen data set, and the spatial capabilities of this space are captured through an initial conformational analysis. This spatial information is compressed and utilized to generate conformational space estimations throughout the algorithm, which expedites the search for solution graphs. The effect of various user determined input parameters is considered and exemplified through a case study involving the identification of solvents falling within a desired boiling point range, as estimated by a multi-dimensional property model.
\end{abstract}

Keywords: Molecular Design, Genetic Algorithm, Descriptors

\section{Introduction}

The area of computer-aided molecular design (CAMD) has greatly influenced the rate and cost at which novel chemicals with desired attributes have been identified. As such, great effort has been invested in new methodologies which allow for the solution of larger and more complex problems of this nature. The application of genetic algorithms (GAs) is one such technique which has shown early promise in the solution of large combinatorial, and highly non-linear molecular design problems (Venkatasubramanian et al., 1995). While there are many established stochastic methodologies capable of solving such problems, studies identifying an optimal approach for such problems have most often proven inconclusive based on the varying nature of individual problems and search spaces (Amaran et al., 2014). It is known, however, that GA's are simple to implement in parallel computing environments, which provides motivation for the development of such an approach that could be scaled up based on the problem size. Genetic algorithms (Holland, 1975) are a subclass of evolutionary algorithms, which mimic the process of natural selection, that encode the characteristics of an individual, in this case a potential candidate molecule, within a chromosome. The typical approach begins with a population of randomly generated individuals and the more fit members, i.e. those closest to possessing the desired characteristics, are selected to undergo computational analogues of natural recombination and mutation. This process is iterative until the resultant population possesses the desired attributes. The nonlinearities under consideration within this paper arise from the utilization of

* To whom correspondence should be addressed: edenmar@auburn.edu 
complex molecular descriptors, which cannot be linearly mapped into chemical space. Additionally, the combinatorial issues stem from the use of molecular fragments as building blocks for developing potential solutions.

Kawai et al. (2014) realized the importance of utilizing a fragment based approach for de novo molecular construction from previous studies in which infeasible molecular structures were regularly created through atom based mutations. Their evolutionary approach to searching for novel chemical structures began with a desirable initial structure, which was evolved by means of mutation and crossover operators while diversity was supplied by a fragment library created from molecules related to the target of interest. This study introduces the challenge of creating chemically feasible molecules, which were in this case verified though a measure of chemical similarity (e.g. Tanimoto coefficient). One limitation however is that the molecular fitness and evolution of structures were guided through this same measure of chemical similarity. It would be beneficial to introduce flexibility in terms of how molecular solutions are ranked, such as the ability to utilize characterization of any dimensionality. Such an approach has proven successful in providing measures of similarity between biologically active molecules (Nettles et al., 2006) as well as chemicals (Tseng et al., 2013). Accessibility and ease of use associated with modern molecular modelling software is allowing the utilization of spatial molecular characterization in a variety of fields. Additionally, combination of these higher order descriptors with the conventional topological and constitutional descriptors of the past is now much more feasible with the application of powerful variable selection techniques (Nicholls et al., 2004).

One of the difficulties arising from the inclusion of spatial descriptors within a property model is that the conformational space, or potential energy surface, of each possible solution must be explored in order to estimate these spatial descriptors. This can become quite a task considering that the degrees of freedom associated with this space is $3 N-6$, with $N$ being the number of atoms in the molecule. Additionally, a molecule will most often have multiple accessible conformations, each of which can correspond to a local energy minimum on the potential energy surface, instead of a single conformation. A combination of these factors makes the consideration of one conformer, often times built from common bond lengths and angles, infeasible for the derivation of spatial descriptors as it is not representative of the set of accessible conformations often seen in bulk chemicals. One recent successful de novo molecular design study (Katarkar et al., 2015) was able to consider the structural, or twodimensional (2D), and spatial, or three-dimensional (3D), properties in an evolutionary approach to discover inhibitors of the Hsp-47 heat shock protein. This study is based upon a previously introduced methodology (Douguet et al., 2005) that uses 3D molecular fragments to generate candidate molecules, which undergo the genetic operators of mutation and crossover to evolve towards an improved fitness. Each candidate molecule must undergo a conformational search in which several conformers are identified through an energy minimization. This step can become computationally intensive, especially for large search spaces, and an approach to estimate the conformational capabilities of candidate molecules within the chemical search space is introduced within the presented methodology. The expedited development of spatial properties is accompanied by a potential decrease in the quality of geometric information. This is the trade off in sacrificing a thorough conformational search for a more computationally efficient method of spatial development. The feasibility of such an approach lies in the sensitivity of the estimated properties/attributes with respect to 
spatial parameters, which may often fall within the error associated with the developed property model. The research gap filled by this methodology resides in the inability of current molecular design approaches to solve problems with multi-dimensional descriptors while considering the conformational space of potential solutions without an extensive conformational analysis performed through molecular simulations. The presented technique is able to estimate the conformational space 'on the fly' for candidate structures, along with facilitating the calculation of topological descriptors through its graph based representation.

The approach presented in this paper utilizes a fragment based descriptor, which is represented as a molecular graph, as building blocks to generate candidate solutions. These building blocks are generated from the set of molecules utilized in creation of the chosen property models such that the resultant region of chemical space searched has an increased likelihood of falling within the applicability domain of these models. The spatial characteristics of this space are captured through an extensive conformational analysis, after which the information generated is compressed to minimize redundancy. This compressed geometric information is then utilized throughout the algorithm to estimate the accessible potential energy surface for the candidate structures considered. Genetic operators of mutation and recombination have been developed to act on this graph based representation of molecules, ultimately guiding the population of candidate structures towards an improved fitness. The objective of this paper is to introduce this methodology and elucidate the effect of some user defined parameters. These effects are exemplified through a case study in which molecules are designed to fall within a certain boiling point range. The authors were invited to submit this paper as an extended version of a paper published in the proceedings of the $24^{\text {th }}$ European Symposium on Computer-Aided Process Engineering (ESCAPE-24) (Herring \& Eden, 2014).

\section{Methodology}

The methodology described herein will begin by extending the concept of signature descriptors to include spatial information. The development of this information through a conformational analysis, along with the subsequent compression of data, will also be discussed. This conformational analysis is necessary to develop spatial information about the molecular fragments, which is useful in estimating the subsequent whole molecule geometric information of candidate solutions. This data is compressed because much of the spatial information is overlapping and would cause combinatorial explosion if left untreated. Once the creation of spatial atomic signatures is introduced, the utilization of these fragments in reproducing molecular geometries representative of a more thorough conformational analysis will be considered with an example illustrating the accuracy and limitations of such a technique. Following this, the genetic algorithm designed to handle this graph based representation of potential solutions will be considered along with details for generation of a starting population, formulation/implementation of genetic operators, and utilization of fitness functions. The novelty of such an approach is that the genetic operators have been design to maintain and perpetuate spatial information within the candidate molecules created. Finally, the effect of some user defined variables will be discussed to help elucidate the decisions for these criteria. The generalized methodology can be visualized in Fig. 1. 


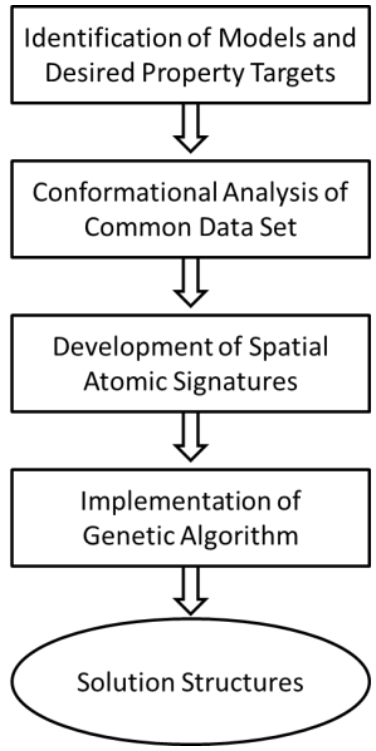

Figure 1: Overview of CAMD methodology developed.

\subsection{Signature Descriptor}

The signature descriptor is a fragment based descriptor which encodes the environment around a central atom up to a pre-defined height, $h$. An example of this is shown below in Fig. 2.

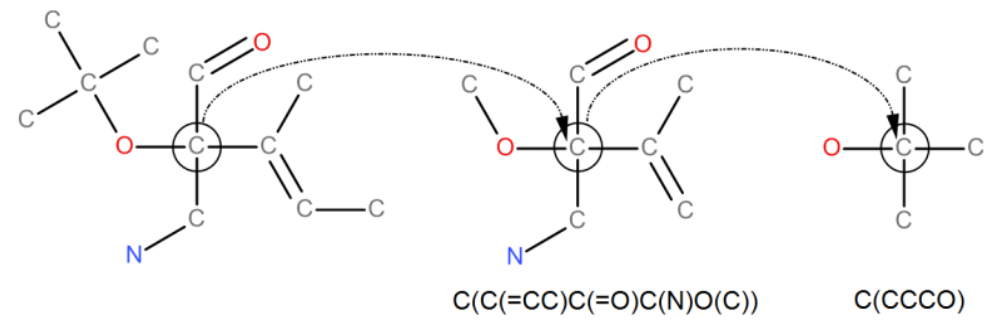

Figure 2: Example of height one and two atomic signatures.

For the structure seen on the left in Fig. 2, the height two atomic signature for the circled carbon atom includes all atoms at a distance of two bonds from this central atom, which is shown in graphical and canonical text format in the middle. In addition, the atoms included in the height one atomic signature, along with the canonical form, are shown on the right. Each atom in the molecule would have its own atomic signature and the summation of these would represent the respective molecular signature. This representation is canonical, which alleviates the pressure of combinatorial explosion since all fragments are uniquely represented. The signature descriptor was originally developed by Visco et al. (2002) and has since proven quite useful in a variety of molecular design applications ranging from the design of HIV-1 protease inhibitors 
(Faulon and Churchwell, 2003) to hydrofluoroether foam blowing agents (Weis et al., 2005).

\subsubsection{Spatial Signature Descriptor}

Most molecular descriptors, from simple constitutional types to more complex topochemical indices, can be derived from molecular signatures (Faulon et al., 2003). This allows one to solve existing structure-activity relationships (SARs) in signature space, while maintaining the predictability of the original SAR along with the low degeneracy attributed to signature descriptors in enumerating potential solutions. However, the original signature representation is limited to capturing only twodimensional information. Extension of the signature descriptor to include spatial information benefits from the efficiencies seen in previous applications while offering the discriminatory power of including descriptors of higher dimensionality in the property models utilized (Herring et al. 2012). This information is developed through molecular mechanical simulations utilized to obtain conformational geometry capabilities of the chemical space under consideration. This spatial data is then stored as a set of conformers, listing the Cartesian coordinates of each atom, for each unique atomic signature. Each atomic signature is then tagged with its own set of conformers, resulting in the respective spatial atomic signature.

\subsubsection{Conformational Analysis}

A set of molecules is chosen for an initial conformational analysis. This set, as visualized in Fig. 3, consists of molecules found in the overlapping data sets of all property models utilized. The candidate molecules generated, from this fragmentized initial data set, will have an increased likelihood of falling within the applicability domain of all models since they represent combinations of the original atomic signatures. The conformational analysis captures the spatial capabilities of the subsequently generated molecular signatures and allows for a quick estimation of their three-dimensional characteristics. The Signature Translator Program (v. 3.0), developed by J.-L. Faulon (2003), is implemented in Unix to calculate the set of canonical atomic signatures found in each conformer. The program accepts as input a .mol formatted file and the desired signature height and returns an output text file containing the respective atomic signatures. NetworkX (Hagberg et al., 2008) is a python language software package containing many modules and functions useful for the graph theoretical analysis of molecules, as well as countless other applications. This software is used to turn each atomic signature into a graph object, which represents the molecular fragment encoded within the signature. Structural isomers are quickly identified, through matching their atomic signatures, and grouped together along with the Cartesian coordinates of each atom found within the structure. While the conformational analysis is quite thorough, the information generated is often redundant. The reason for this is that each atomic signature can often be found in various molecules, each having a different observed environment outside of the prescribed signature height. The conformers developed from each of these structural isomers are bound to have overlapping information, which necessitates compression of this data to avoid subsequent combinatorial issues. 


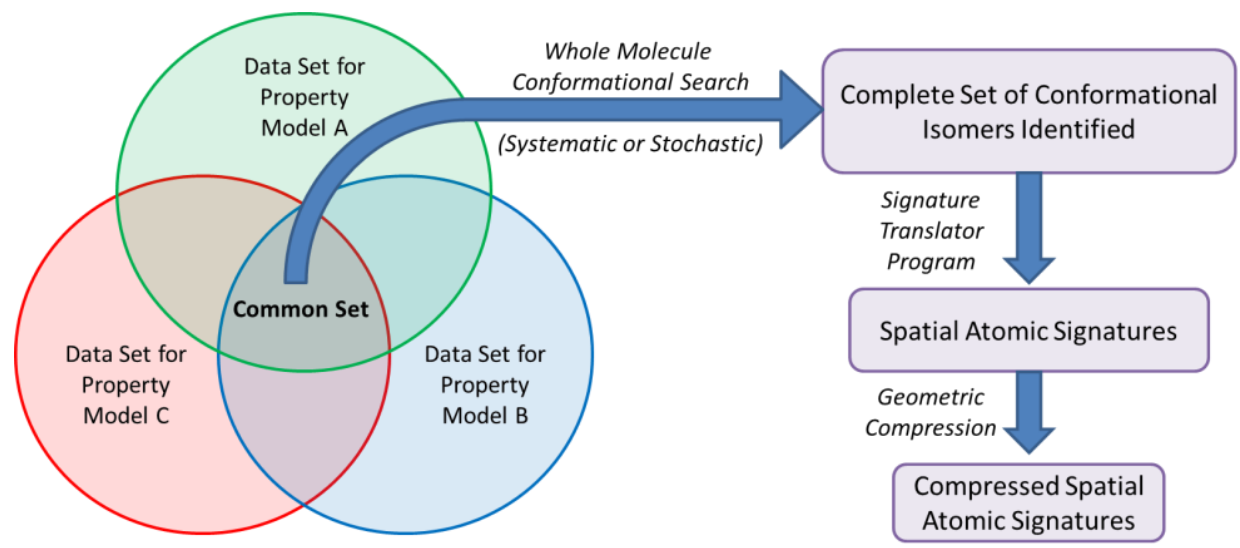

Figure 3: Algorithm visualization for development of spatial signatures from original property model data sets.

Each unique atomic signature, corresponding to a structural isomer, will have its own set of conformers identified during the conformational analysis. However, much of this information is redundant so they must be reduced so that only the minimal number of conformers is necessary to capture the spatial capabilities of each fragment. To do this, each pair of conformers for an atomic signature is compared based upon the interatomic distances found within the structures. A cutoff value is established and utilized as a threshold for comparison, beyond which both conformers would be kept because they both capture a unique conformation. However, when all interatomic distances among both conformers fall below the cutoff, one is discarded at random because of the high degree of similarity. This technique allows flexibility in the degree of compression through variance in the chosen cutoff value. The effect of varying this cutoff value for a set of 24 common industrial solvents is shown in Table 1. These solvents were dissected into 73 unique atomic signatures such that the pre-compression average number of conformers per signature was 5.3, and was reduced to around 2.5 with a cutoff value of 2 Angstrom.

Table 1: Compression changes with varying cutoff values.

\begin{tabular}{|c|c|}
\hline $\begin{array}{c}\text { Cutoff Criterion } \\
\text { (Angstrom) }\end{array}$ & $\begin{array}{c}\text { Conformers } \\
\text { Remaining }\end{array}$ \\
\hline 0 & 387 \\
\hline 0.05 & 282 \\
\hline 0.10 & 271 \\
\hline 0.20 & 257 \\
\hline 0.30 & 240 \\
\hline 1.00 & 199 \\
\hline 2.00 & 182 \\
\hline
\end{tabular}

\subsubsection{Geometry Development Verification}

The technique developed within this methodology requires the estimation of wholestructure molecular geometries from individual fragment geometries. While convenient, the feasibility of such an approach must be verified for application within a molecular 
design framework requiring accurate spatial information. The ideal approach for developing geometry information for each structure under consideration would be to perform a complete systematic conformational analysis of the full molecule. However, since this approach is currently too computationally demanding for a considerable search space, the initially proposed methodology has been developed. The technique chosen for verification of this methodology is to create a set of spatial atomic signatures with various compression values and use these in an attempt to recreate conformational isomers identified for a test set, which was not included within the initial data set. The ability to recreate the conformers for these test set molecules, which were analyzed as whole structures, will serve as verification of the proposed methodology. The data set chosen for this task includes: n-pentane, n-heptane, 2-hexene, 2,5-heptene, 2-methylheptane and 2,5-dimethylhexane. The test set, for which it is desired to reproduce geometric information from the data set analysis, consists of n-hexane, 2,5-hexene and 2-methylhexane.

For the test set molecules, there were 7 conformers identified for $n$-hexane, 5 conformers for 2,5-hexene and 9 conformers for 2-methylhexane. Based upon the chosen data set for this example, all conformers were able to be recreated with a compression cutoff value of 5.5 Angstrom and a conformer comparison cutoff of 3.2 Angstrom. The conformer comparison value determined the degree of similarity necessary for two conformers to be considered as capturing the same spatial information. This example was a success in that all of the desired conformers were created; however, the identification of a reasonable compression value is a crucial step in the design algorithm.

\subsection{Genetic Algorithm Developed}

The genetic algorithm applied within this methodology is represented in Fig. 4. This process begins with the development of spatial atomic signatures, which was discussed in Section 2.1. The generation of a starting population is necessary to begin the evolutionary loop of selecting and operating on individual members. The fitness of each of these candidate structures is estimated through a carefully chosen fitness function, which captures 'closeness' to the desired attributes. Graphs are chosen on which to apply genetic operators, which ultimately evolve successive generations towards an improved overall fitness. These operators are categorized as either mutation or crossover and their implementation and effects are discussed below. An end point is reached based upon various criteria such as the overall fitness of a given population reaching a desired value or a specific number of generations passing. Solutions are identified throughout the algorithm and stored for later display. The generation of feasible molecular structures is expedited through utilization of what has been termed a 'bonding network'. The creation and utilization of this network is discussed in section 2.2.1. Further, the conventional use of signature descriptors is to develop a molecular signature consistent with certain graphicality and consistency equations (Visco et al., 2002) and ultimately derive one or more structural isomers that are represented by this set. The enumeration of all structural isomers, being the most computationally intensive step within such an approach, is bypassed in this methodology. While convenient for a systematic search through a region of chemical space, signature descriptors are overdefined in such a manner that only a portion of the atomic signatures from a molecular signature are utilized in the development of geometric features. These are the fragments that are identified throughout the implementation of this evolutionary algorithm. As such, the computational expense of a systematic search for structural isomers, as done in 
previous CAMD applications (Herring et al., 2013) involving signature descriptors, is omitted in exchange for a more stochastic approach in this paper.

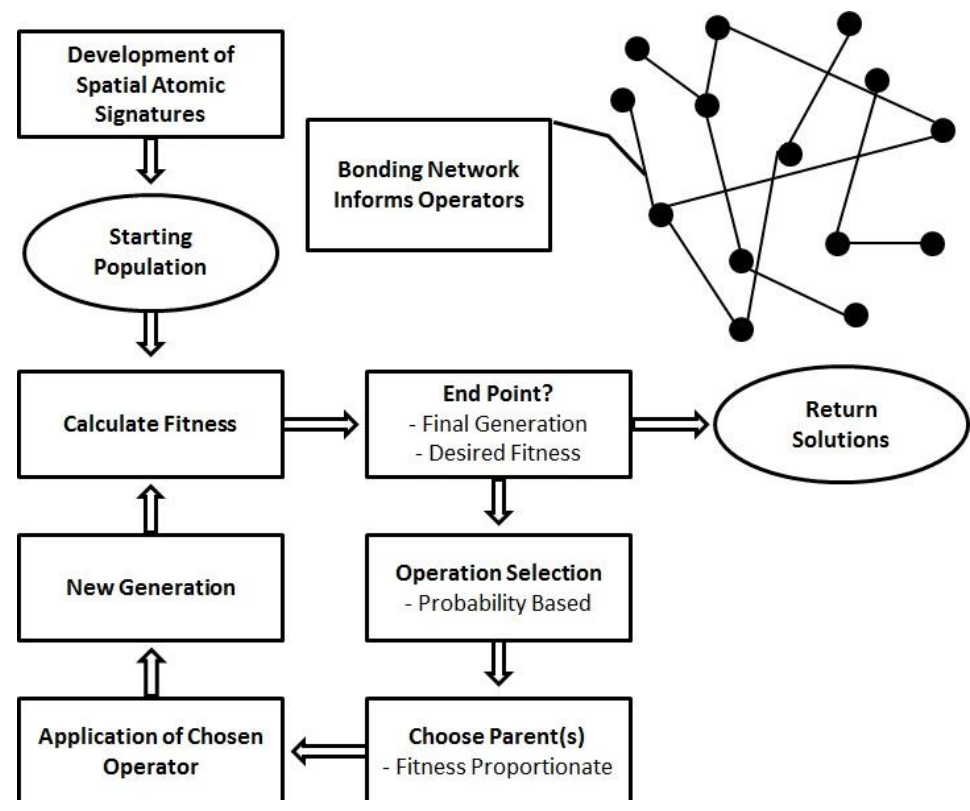

Figure 4: Flowchart for genetic algorithm developed.

\subsubsection{Bonding Network}

Since atomic signatures capture the environment, up to a selected distance away, around a central atom, the signatures describing a molecule will overlap by design. The overlap is taken advantage of in the development of structural and conformational isomers. The generation of structural isomers throughout this methodology requires knowledge of the potential overlap between all atomic signatures involved. This information is precalculated and stored within a network, referred to as the 'bonding network', for subsequent use. NetworkX is utilized for this task of creating a network which stores the overlap capabilities of each atomic signature with respect to other signatures generated from the original data set. Each unique atomic signature is initiated as a node within this 'bonding network', which initially contains no edges. Once the network has been compressed to contain a minimal amount of conformers capturing the original spatial diversity, nodes are analyzed pairwise to establish possible bonds. A bond between two atomic signatures, ${ }^{h} \sigma_{M}(x)$ and ${ }^{h} \sigma_{M}(y)$, is possible when the ${ }^{h-1} \sigma_{M}(z)$ signature of atom $z$, neighboring atom $x$, matches the ${ }^{h-1} \sigma_{M}(y)$ signature. This can also be stated, from a graph theoretical viewpoint, as a subgraph, with radius $h-1$ centered at an atom neighboring the $x$-signature center atom, being isomorphic to the radius $h$ - 1 subgraph centered at the $y$-signature center atom. Additionally, a visualization of this constraint can be seen in Fig. 5. 

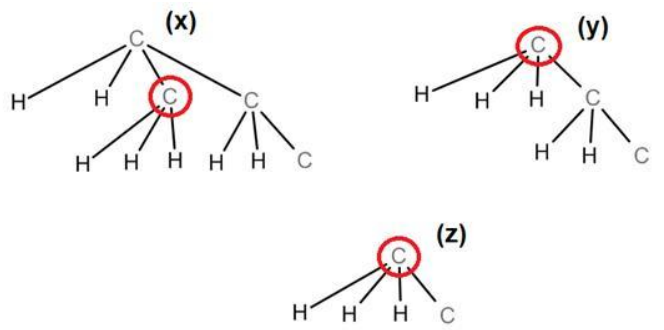

Figure 5: Example of bond compatible atomic signatures. The two atomic signatures centered at atoms (x) and (y) are capable of bond formation within the network since they possess the common subgraph shown in (z).

To facilitate subsequent docking operations, used to establish a global geometry between the potentially discrepant fragment geometries, a mapping dictionary is stored within each edge. This dictionary correlates the overlapping atoms, which each have their own unique node labels within the two signatures. Additionally, there is often more than one possible mapping and each of these are stored as well. An optimal mapping would contain three common atoms, while maximizing the amount of unmapped atoms. This ensures that a maximal amount of geometric information is developed per docking operation within the molecule, since the amount of overlapping geometry data is minimized and unique geometry data is maximized. Pseudo-code has been provided within Appendix A, along with tips for utilizing NetworkX, for the implementation of such a procedure.

\subsubsection{Starting Population}

An initial generation of molecules is selected at random to develop a population, which encompasses a variety of structural features spanning the chosen chemical space, on which to begin reproduction. The input required for this step consists of an upper and lower limit on atom count as well as a population size. For each member of the chosen population size, a target atom count is selected at random from the acceptable size range. A node is then selected at random, from the bonding network, to begin the graph and the possible neighbor list, from which the subsequent overlapping signature fragment is chosen, is developed from information contained within the bonding network. This process continues for a growing graph while the selection of signature fragments is a function of the current graph size. Eq. (1) is utilized as a probability function for the selection of a signature which would either maintain graph unsaturation or effectively cap the growing molecule when nearing the desired size. In this equation, $P_{c a p}$ is the probably of selecting a signature fragment which would saturate the growing graph, $N_{T}$ is the target number of atoms and $N_{C}$ is the current number of atoms.

$P_{c a p}=1-\left[\frac{\left(N_{T}-N_{C}\right)}{N_{T}}\right]$

It is possible for the probability to exceed a value of one, in which case graph growth would be immediately terminated. Also, based upon the data set under consideration, it might not be possible to immediately cap a graph or continue its growth because of the potential unavailability of fragments facilitating this operation. This approach helps provide a range of graph sizes for the starting population, while still contained within a 
desired range. The selection of a desired size range allows for the inclusion of some heuristic knowledge within the design algorithm and the generation of a starting population can be visualized in Fig. 6.

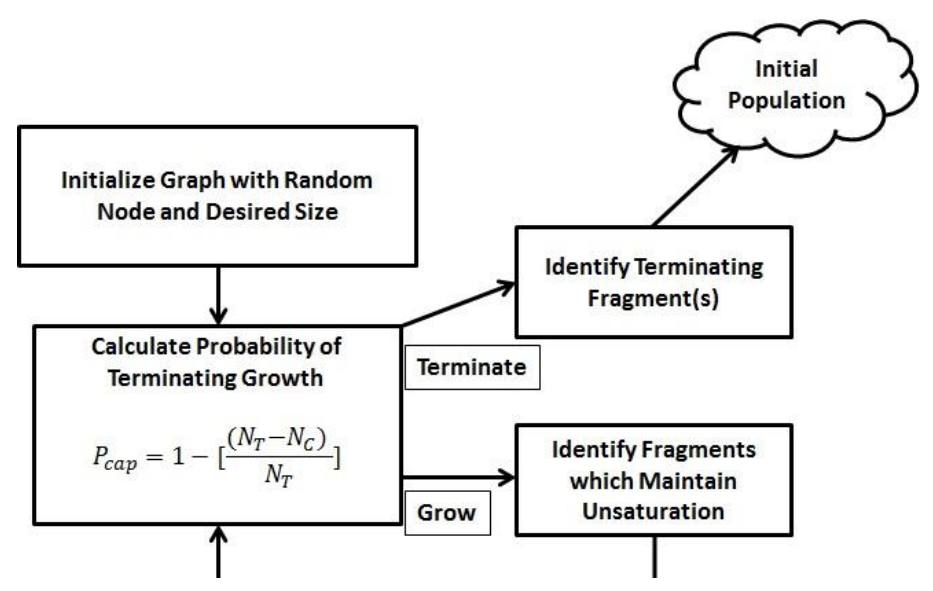

Figure 6: Flowchart or development of initial population.

The concept of 'growing' a molecular graph from overlapping fragments defined within the bonding network is visualized below in Fig. 7. An example is provided for the growth of a molecular graph being developed for use within the initial population.

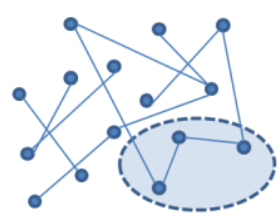

Bonding Network

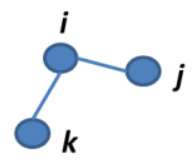

$\underline{\text { Initiated Subgraph }}$

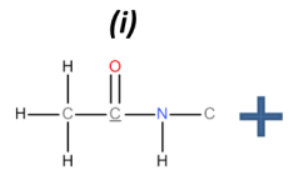

(i)

\section{(j)}<smiles>CNCC1CCCC1</smiles>

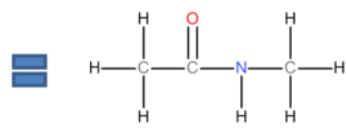

(k)
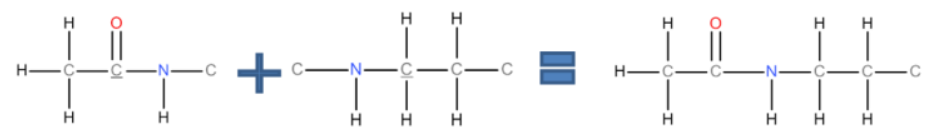

Figure 7: Visual example of 'growing' a member of the starting population, which was initialized with node $i$, from information within the bonding network. The root atom for each atomic signature has been underlined within the example provided. 
Within Fig. 7, node $i$ was randomly chosen to initialize a graph for inclusion within the starting population. Based upon information within the bonding network, two nodes $(j$ and $k$ ) are identified as having a common subgraph with the fragment represented by node $i$. Based upon the desired size of this graph, the decision to terminate growth or perpetuate unsaturation is available with the selection of node $j$ or $k$, respectively. This example utilizes height two atomic signatures, whereas smaller fragments could also be implemented with a lower height. However, smaller fragments could result in poorly estimated geometries and necessitate more coordinate transformation calculations.

\subsubsection{Fitness Function}

The fitness function provides a measure of how close a candidate is to satisfying the chosen properties/attributes of interest. This metric is necessary for the selection of candidate structures on which genetic operators will be applied and is meant to mimic the pressure of natural selection. The function chosen for such a task has been applied in previous molecular design problems (Venkatasubramanian et al., 1995) with success and its form is shown in Eq. (2).

$f_{i}=\exp \left(-\alpha\left[\sum_{i}^{n} \frac{\left(P_{i}-P_{i, b a r}\right)^{2}}{\left(P_{i, \text { max }}-P_{i, \text { min }}\right)^{2}}\right]\right)$

This format is well suited for application within molecular design problems, which often require candidate solutions to fall within an acceptable property range or a set of property ranges. These ranges are established with $P_{i, \max }$ and $P_{i, \min }$, which represent the desired upper and lower bounds for a given property, respectively. With $P_{i, \text { bar }}$ being the average between this upper and lower limit, this formulation allows the fitness to be expressed as a smooth Gaussian function with values ranging from zero to one. In this case, $\alpha$ represents a tunable parameter known as the Gaussian decay rate, which controls how quickly the fitness value falls off as the property values leave the desired region. This effect can be visualized in Fig. 8.

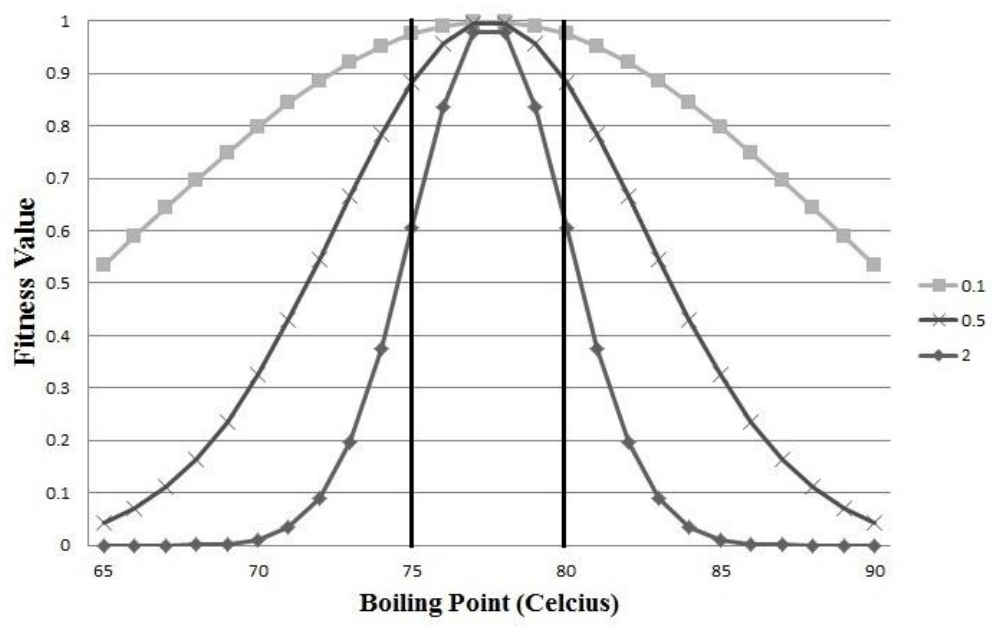

Figure 8: Effect of $\alpha$, Gaussian decay rate variable, on resultant fitness over a desired property range. 
From Fig. 8, one can imagine how $\alpha$ can be utilized to control the 'strictness' of the constraints based upon the size of a given property range. The example provided within this figure depicts the effect of varying the $\alpha$ value, ranging from 0.1 to 2 , will have on how closely a candidate structure must be to the desired property range, which is between 75 and $80{ }^{\circ} \mathrm{C}$ in this case. It can be seen that decreasing $\alpha$ will allow for properties falling outside the desired region to have a higher fitness value when compared to fitness functions utilizing a larger $\alpha$ value. This can be taken advantage of in a CAMD application by allowing one to tune this parameter in response to how heavily populated the search space is with solutions falling within the desired property range. A search space with many near optimal solutions can be more stringently searched by placing a larger $\alpha$ value, whereas less optimal solutions could be considered with a decreased $\alpha$ value in a space sparsely populated with reasonable solutions.

\subsubsection{Genetic Operators}

Various genetic operators are applied to maintain diversity within the population and effectively explore the available chemical space. New techniques were developed to tackle the unique format of the problem addressed here, namely the evolutionary development of graph-based molecular representations. The two main types of operators are mutation and crossover. Within the category of mutation operators, three types were created: reduction, insertion, and fragment mutation. The probability based decision of which mutation operator to utilize is a function of the current graph size, in addition to a parameter, $N_{o p t}$, which represents the current optimal graph size derived from previous data. $N_{\text {opt }}$ is continuously updated as generations are analyzed and is calculated as shown in Eq. (3).

$N_{o p t}=\frac{\sum_{i}\left(f_{i} \cdot N_{i}\right)}{\sum_{i} f_{i}}$

Eq.(3) represents the optimal size as a function of fitness $\left(f_{i}\right)$ and atom count $\left(N_{i}\right)$ for all molecular graphs $(i)$ analyzed up to that point. The selection of a mutation or crossover operation is a probabilistic event, which is discussed more thoroughly within Appendix A. Once this decision has been made, the parent(s) for the given operation must be chosen. This step is performed using a standard fitness proportionate selection technique where the probability of being selected is based on the relative fitness of the individual molecular graphs. Mutation operations require only one selection whereas crossover operations need two members to be chosen from the current population.

Once a mutation type operation has been decided upon, the selection of which operator is a function of the current graph size and the optimal graph size $N_{o p t}$, referenced in Eq.(2). Graphs within a certain percentage $\beta$ of the optimal size undergo a signature mutation such that the size of the graph is preserved, while graphs above and below this range undergo deletion and insertion, respectively. This approach is exemplified in Fig. 9 where a molecule described by two overlapping atomic signatures undergoes each of the described operations. 


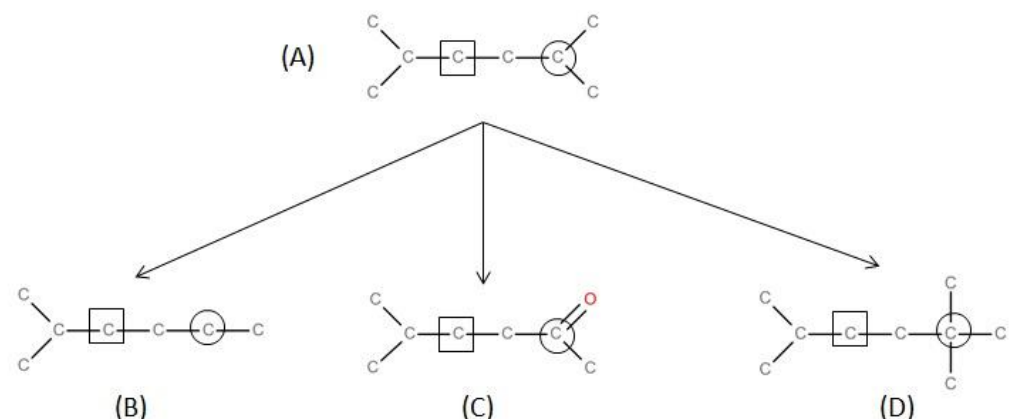

(B)

(C)

(D)

Figure 9: Mutation operators. Molecular graph (A) undergoes a deletion mutation on circled signature and produces graph (B). Similarly, graphs (C) and (D) are produced through a signature mutation and insertion mutation, respectively.

Signature mutation involves the selection of a random node, which is then exchanged, based on bond compatibility information available in the bonding network, preserving the original size. Graph reduction involves the selection of a random signature within the graph, which is then replaced by a smaller signature identified within the bonding network. Similarly, graph insertion involves the selection of a random signature, which is replaced by a larger signature, meeting the desired bonding capabilities as defined within the bonding network, to increase the size of the resultant molecular graph. If the signature selected does not have a suitable replacement, resulting in the desired mutation, other signatures within the molecule are considered. Ultimately, if no feasible mutations are identified within the bonding network then another member of the population is chosen for alteration through the steps discussed above.

Upon selection of the crossover operator, two parent graphs are selected from the population based upon recombination compatibility and fitness values. Details of the fitness proportionate selection of two parents, compatible of a crossover operation, are discussed within Appendix A. At least three common atoms are necessary, within the isomorphism mapping between the two graphs chosen for crossover, for the development of molecular geometry information. For this study, only single-point crossovers were utilized and this approach is visualized in Fig. 10.

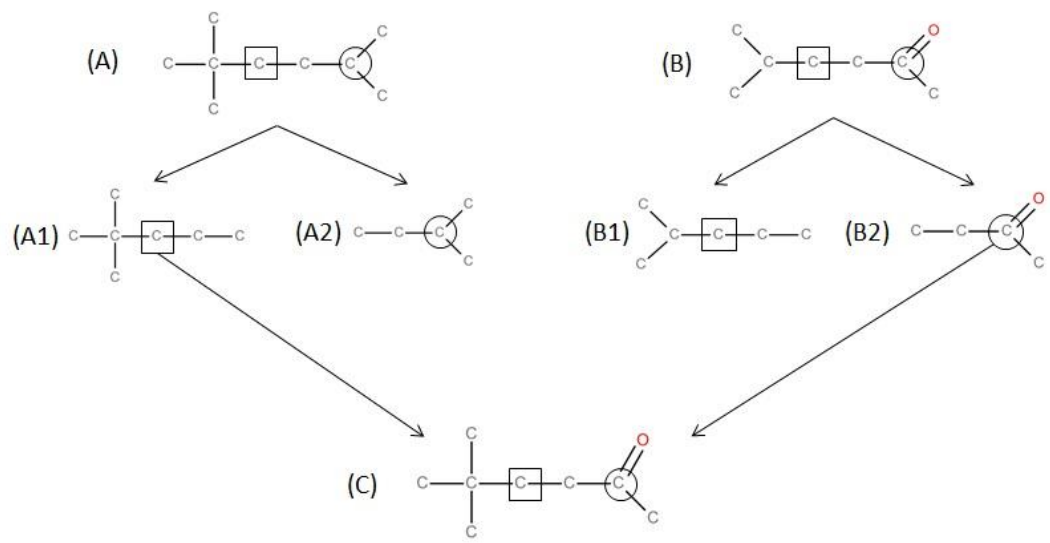


Figure 10: Example of crossover operator. An atomic signature (A1) from graph (A) can be overlapped with an atomic signature (B2) from graph (B) to form a new candidate graph $(\mathrm{C})$.

Each of these operators rely heavily on information stored within the bonding network, which is why this information is pre-calculated for the signature space developed from the initial data set. Additionally, the selection of these operators is based upon the optimal size identified throughout the algorithm. In cases where the optimal solutions might not be a strong function of molecular size, the selection of operators could be held independent of this information by deciding a probability value for each operator irrespective of the current or optimal size. While the mutation operators were specifically designed to guide graph towards an acceptable size region, the crossover operator allows for the consideration of molecules that might fall just outside this area. As such, the effect of the probability based decision between mutation and crossover operators has an effect on the size distribution of the resultant population, which is exemplified in Fig. 11.

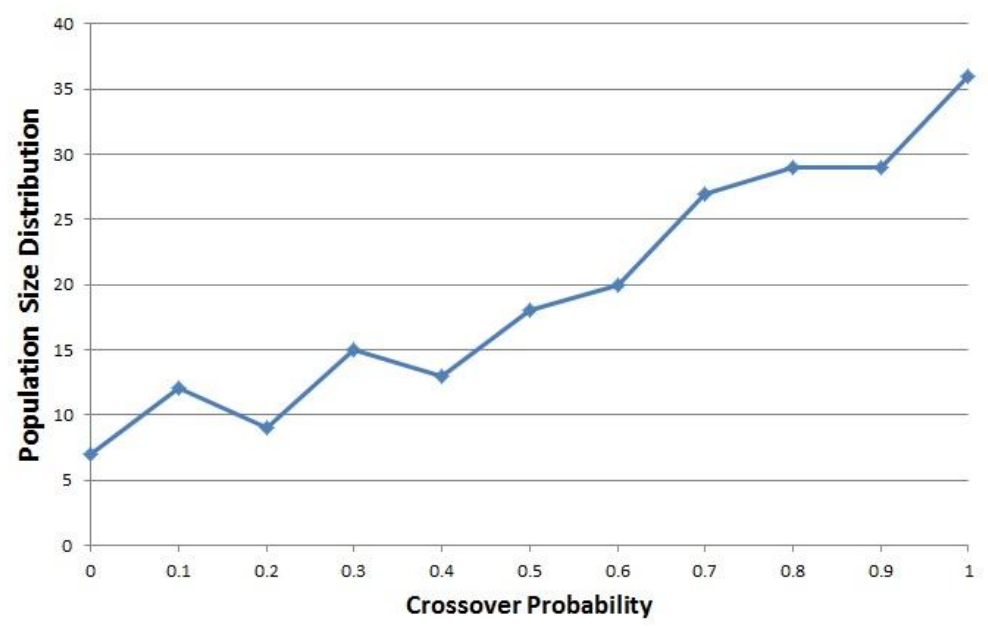

Figure 11: Effect of crossover probability on the population size distribution.

From Fig. 11, it can be seen that an increase in the probability of selecting a crossover operator results in a wider distribution of molecular graph sizes. The population size distribution in this example represents the difference between the largest and smallest atom count after 10 generations for a population of 30, while applying a $\beta$ value of 0.15 .

\subsubsection{Conformational Analysis}

Once the graphs for each member of the population are developed, their underlying geometry is developed by uniting the fragment geometries into a consistent frame of reference. The previously established (Herring et al., 2013) requirement for overlapping signatures is that they share at least three atoms in common and these atoms are used to transform the coordinates. There are three steps necessary for transformation and they include an initial translation followed by two rotations. The initial translation is facilitated through a vector, created between two mapped atoms within the signatures. These initial atoms are paired with two more to provide an axis of rotation which ensures that both signatures now have a common axis. The final step is a rotation 
around this common axis to minimize deviation between the third, or any remaining, mapped atoms. A visualization of these concepts is provided in Fig. 12.

(A)

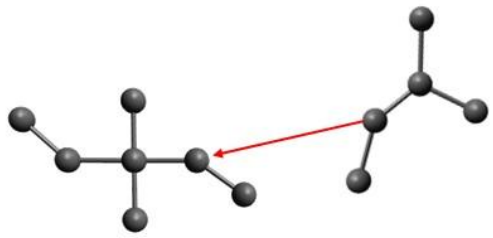

(B)

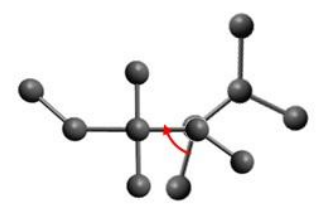

(C)

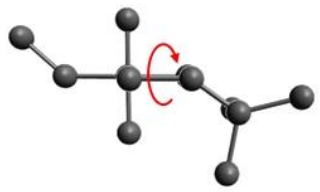

Figure 12: Docking of two atomic signature fragments where (A) depicts the initial transformation, (B) depicts the rotation to develop a common axis and (C) depicts the final rotation to minimize difference in coordinates.

This information can be stored and applied with a transformation matrix, which is shown below, for the rotations, in Eq. (4). Within Eq. (4), $u$ represents the axis of rotation while $\theta$ represents the angle of rotation. The collective movement associated with a given transformation is developed as the product of the two rotation matrices and the homogeneous translation matrix. Each atomic signature within the graph has a set of accessible conformations, which are utilized in an iterative manner to develop a conformational ensemble for each molecular graph considered. The topographic descriptors are averaged over this ensemble to account for variations seen in geometries throughout the conformational space.

$$
R=\begin{array}{ccc}
\cos \theta+u_{x}^{2}(1-\cos \theta) & u_{x} u_{y}(1-\cos \theta)-u_{z} \sin \theta & u_{x} u_{z}(1-\cos \theta)-u_{y} \sin \theta \\
u_{y} u_{x}(1-\cos \theta)+u_{z} \sin \theta & \cos \theta+u_{y}^{2}(1-\cos \theta) & u_{y} u_{z}(1-\cos \theta)-u_{x} \sin \theta \\
u_{z} u_{x}(1-\cos \theta)+u_{y} \sin \theta & u_{z} u_{y}(1-\cos \theta)+u_{z} \sin \theta & \cos \theta+u_{z}^{2}(1-\cos \theta)
\end{array}
$$

\section{Case Study}

The case study chosen to exemplify this approach involves the design of a molecule with a specified boiling point temperature. Basak et al. (1996) developed a structure activity relationship correlating various $2 \mathrm{D}$ and $3 \mathrm{D}$ molecular descriptors to the normal boiling point for a data set of 1023 chemicals from the Toxic Substances Control Act (TSCA) Inventory. The best fit model, shown in Eq.(4), resulted from a combination of 2D topological, 2D topochemical, and 3D descriptors with an $\mathrm{R}^{2}$ of 0.967 . 
$B P=-287.5+125.3\left(\chi^{6}\right)+10.9\left(P_{10}\right)+74.5\left(I C_{0}\right)-125\left(\chi_{b}^{6}\right)-86.3\left(\chi_{C b}^{3}\right)+$ $175.3\left(\chi_{v}^{0}\right)+49.1\left(\chi_{v}^{2}\right)+18.7\left(\chi_{P C v}^{5}\right)-9.1\left(W_{3 D}^{H}\right)+8.1\left(W_{3 D}\right)$

Within the model, it can be seen that there is a wide variety of molecular descriptors that have been deemed optimal, in combination, at modelling the boiling points of these chosen chemicals. These descriptors include topological, topochemical, geometric, and information theoretic indices. As such, it represents an exemplary model for application of the proposed molecular design methodology.

\subsection{Spatial Signatures from Data Set}

A subset of 245 molecules was chosen from the initial 1023 utilized to develop the boiling point property model with the aim of maintaining the original variance in structural features. A conformational analysis was performed for these molecules with an acceptance criterion of each conformer being within $15 \mathrm{kcal} / \mathrm{mol}$ of the identified conformational minimum. The conformational studies were performed in the Biochemical and Organic Simulation Software (BOSS) (Jorgensen \& Tirado-Reeves, 2005) utilizing a Monte Carlo conformational search with 100 starting structures. These conformers were dissected into 194 unique height-2 atomic signatures, or structural isomers. The conformational information for each signature was further compressed by removing conformers exhibiting a similarity limited to 0.2 angstrom for each pairwise inter-atomic distance comparison, which left 582 spatial signatures.

\subsection{Parameters Chosen}

The parameters necessary for this design problem include a lower (10) and upper (25) bound placed on the number of atoms allowed in the generation of an initial population. These bounds were large enough to consider molecular graphs representative of solutions falling within the desired property range. In addition the lower and upper bounds for boiling point were placed at 75 and $80{ }^{\circ} \mathrm{C}$, respectively. An initial population size was set at 500 while the steady state population size was chosen to be 75 graphs and each run, of which there were 10 total, continued for 50 generations. The probability of selecting a crossover operation was set to 0.3 based upon initial studies with the criteria of maintaining the original size distribution set for the initial population. The $\beta$ variable discussed in section 2.3.2 was set to 0.15 and the Gaussian fitness decay rate, $\alpha$, was set to 1.0 based upon the small desired property range, as exemplified in Fig. 8.

\subsection{Results}

Each run was able to identify several solutions to the problem at hand. The average number of total solutions identified was $29.8 \pm 3.7$, while several solutions were visited more than once, leaving the average number of unique solutions at $22.1 \pm 4.2$. Based upon the frequency of solutions visited more than once, future studies will likely consider the size of the search space during selection of the population size and number of generations. The algorithm was successful at improving the average fitness of each population through successive generations by applying the proposed genetic operators. This effect is visualized in Fig. 13, where the results from all ten runs have been averaged. 


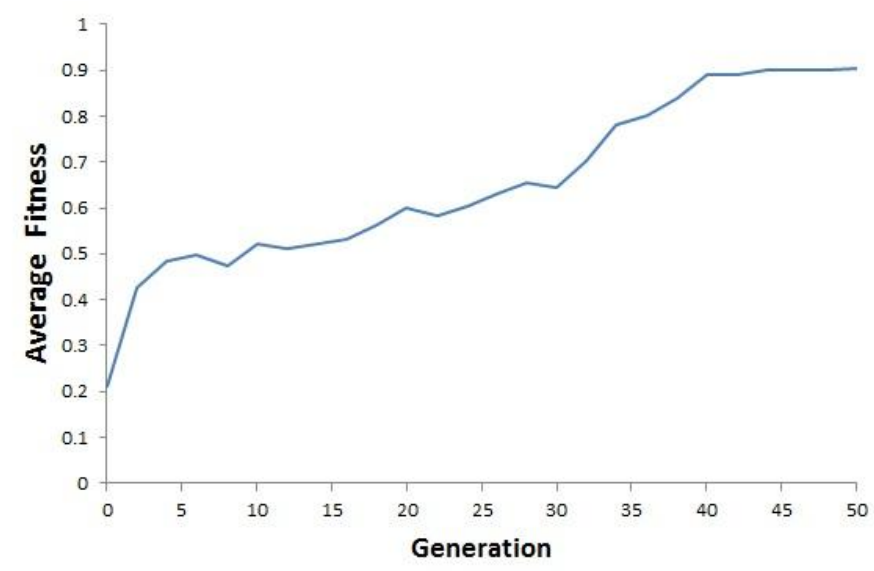

Figure 13: Fitness as a function of generation number.

From Fig. 13 it can be seen that the fitness reaches a plateau, beyond which the average fitness of a future population is less likely to improve. It could be beneficial to the algorithm to include a criterion for termination arising when the change in average fitness of a series of populations reaches zero. Some of the recurring solutions exhibiting a higher degree of fitness have been identified in Table 2. These solutions also provided the closest match to experimental data, whereas there were many structures with poorly estimated property values. This can be attributed to error within the property model chosen as well as potentially poorly estimated geometries. Further investigations of the ability of the methodology to reproduce accurate geometries for a wide range of structures are necessary.

Table 2: Best solutions identified within case study.

\begin{tabular}{|l|c|c|}
\hline \multicolumn{1}{|c|}{ Solution } & Predicted Boiling Point $\left({ }^{\circ} \mathbf{C}\right)$ & Fitness Value \\
\hline ethyl acetate & 77.3 & 0.99 \\
\hline 2,2-dimethylpentane & 77.8 & 0.99 \\
\hline isopropyl alcohol & 78.0 & 0.99 \\
\hline 3,4-dimethyl-1-pentene & 78.1 & 0.98 \\
\hline 2,3-dimethyl-2-butene & 76.7 & 0.97 \\
\hline 2,3,3-trimethyl-1-butene & 78.3 & 0.97 \\
\hline 2-methyl-1,3-pentadiene & 76.4 & 0.95 \\
\hline Ethanol & 78.7 & 0.94 \\
\hline
\end{tabular}




\begin{tabular}{|l|c|c|}
\hline 1,3,5-hexatriene & 78.8 & 0.93 \\
\hline carbon tetrachloride & 75.9 & 0.90 \\
\hline 2,2,2-trifluoroethanol & 79.3 & 0.87 \\
\hline 4,4-dimethyl-2-pentene & 79.4 & 0.86 \\
\hline 2-butanone & 79.5 & 0.85 \\
\hline 2-methyl-2-propanol & 79.5 & 0.85 \\
\hline 1-butanal & 75.5 & 0.85 \\
\hline Acetonitrile & 79.7 & 0.82 \\
\hline 1,2-hexadiene & 75.0 & 0.77 \\
\hline 2,2,3-trimethylbutane & 80.0 & 0.77 \\
\hline 2,4-dimethylpentane & 80.1 & 0.76 \\
\hline
\end{tabular}

\section{Conclusions}

A methodology for the guided stochastic, de novo generation of molecules exhibiting desired properties and attributes has been presented. This approach applies the evolutionary search heuristic known as a genetic algorithm and allows the consideration of many different types of molecular characterization through adaptation of atomic signature descriptors. Developed with the ability to capture graph theoretical descriptors in a molecular design context, atomic signature descriptors have been extended to include spatial information. This results in a powerful technique useful for solving molecular design problems with widely varying descriptor types and property model formats.

Additionally, the nature of genetic algorithms allows for an efficient, guided search through large regions of chemical space. The exhaustive combinatorial search for solutions in a chemical space of this size would have been much more time consuming, whereas the genetic algorithm applied here was able to identify satisfactory solutions, verified through experimental data, much more quickly. A deterministic approach towards analyzing all possible collections of fragments based on this data set would have required the consideration of at least 170,000 potential solutions, based on a conservative estimate of two fragments per molecule. Additionally, each of these structural isomers would have multiple conformational isomers that needed to be identified. The stochastic approach towards identifying structural isomers, along with the techniques developed for estimating the accessible conformational space of these isomers allows for a significant decrease in the computational time necessary to identify feasible solutions. A total of 4,250 molecules were analyzed for each run, which represents a significantly reduced search space when compared to the deterministic approach. A vast majority of this space was avoided through utilization of the bonding 
network, which established feasible collections of overlapping fragments. Simulations have shown that the average time taken to estimate the conformational space for the chosen data set was around 5 seconds per molecule. Comparatively, a Monte Carlo conformational search on the same data set took between 9.3 and 38.9 seconds per molecule for 100 and 500 starting structures, respectively. Coupled with the reduced search space, when compared to a deterministic approach, this data reveals the significant reduction in computational requirements afforded by the methodology developed.

This approach could benefit from inclusion of new types of mutation operators; however, the techniques utilized here were effective in controlling the size and diversity of solutions generated. There is still much work to be conducted in order to analyze the sensitivity of this approach with respect to the various parameters utilized. Future work will likely include a thorough analysis of these parameters and their effect on the run time as well as the diversity and magnitude of solutions generated. In addition, studies with a more justifiable inclusion of conformational data, such as the design of inhibitors satisfying a pharmacophore model, should be considered. However, this case study was suitable for confirmation of the proposed methodology because of the availability of experimental data in verifying the solutions generated. In conclusion, this algorithm represents a novel technique useful for the estimation of conformational space data utilized in solving computer-aided molecular design problems requiring property models with descriptors of varying dimensionality.

\section{Acknowledgments}

The authors greatly appreciate the financial support for this work provided by the US National Science Foundation through the Auburn University Integrative Graduate Research and Education Traineeship (IGERT) program (Award\# 1069004) and the US Department of Agriculture National Institute of Food and Agriculture (Award\# 2011 67009-20077).

\section{References}

S. Amaran, N. Sahinidis, B. Sharda, S. Bury, 2014, Simulation optimization: a review of algorithms and applications, 4OR, 12(4), 301-333.

S. Basak, B. Gute, G. Grunwald, 1996, A Comparative Study of Topological and Geometrical Parameters in Estimating Normal Boiling Point and Octanol/Water Partition Coefficient, J. Chem. Inf. Comput. Sci., 36, 1054-1060.

D. Douguet, H. Munier-Lehmann, G. Labesse, S. Pochet, 2005, LEA3D: A Computer-Aided Ligand Design for Structure-Based Drug Design, Journal of Medicinal Chemistry, 48, 2457 2468.

J.-L. Faulon and C. Churchwell, 2003, The Signature Molecular Descriptor . 2. Enumerating Molecules from Their Extended Valence Sequences, J. Chem. Inf. Comput. Sci., 43, 721-734.

J.-L. Faulon, D. Visco, R. Pophale, 2003, The Signature Molecular Descriptor. 1. Using Extended Valence Sequences in QSAR and QSPR Studies, Journal of Chemical Information and Computer Sciences, 43, 707-720.

A. Hagberg, D. Schult, P. Swart, 2008, Exploring network structure, dynamics, and function using NetworkX, Proceedings of the $7^{\text {th }}$ Python in Science Conference, 11-15.

R. Herring, R. Namikis, N.G. Chemmangattuvalappil, C.B. Roberts, M.R. Eden, 2012, Molecular Design Using Three-Dimensional Signature Descriptors, Computer-Aided Chemical Engineering, 31, 225-229.

R. Herring, J.C. Haser, S. Hada, M.R. Eden, 2013, Structure Based Design of Non-Peptide Mimetics, Computer Aided Chemical Engineering, 32, 175-180. 
R. Herring and M.R. Eden, 2014, De Novo Molecular Design using a Graph-Based Genetic Algorithm, Computer Aided Chemical Engineering, 33, 7-12.

J. Holland, 1975, Adaptation in Natural and Artificial Systems, The University of Michigan Press, Ann Arbor.

W. Jorgensen and J. Tirado-Rives, 2005, Molecular modeling of organic and biomolecular systems using BOSS and MCPRO, Journal of Computational Chemistry, 1689-1700.

A. Katarkar, P. Haldar, K. Chaudhuri, 2015, De novo design based pharmacophore query generation and virtual screening for the discovery of Hsp-47 inhibitors, Biochemical and Biophysical Research Communications, 456, 707-713.

K. Kawai, N. Nagata, Y. Takahashi, 2014, De Novo Design of Drug-Like Molecules by a Fragment-Based Molecular Evolutionary Approach, Journal of Chemical Information and Modeling, 54, 49-56.

J. Nettles, J. Jenkins, A. Bender, Z. Deng, J. Davies, M. Glick, 2006, Bridging Chemical and Biological Space: "Target Fishing" Using 2D and 3D Molecular Descriptors, J. Med. Chem., 49, 6802-6810.

A. Nicholls, N. MacCuish and J. MacCuish, 2004, Variable selection and model validation of 2D and 3D molecular descriptors. Journal of computer-aided molecular design, 451-474.

Y. Tseng, A. Hopfinger, E. Esposito, 2012, The great descriptor melting pot: mixing descriptors for the common good of QSAR models, J. Computer Aided Mol. Design, 26, 39-43.

V. Venkatasubramanian, K. Chan, J. Caruthers , 1995, Evolutionary Design of Molecules with Desired Properties Using the Genetic Algorithm, J. Chem. Inf. Comp. Sci., 35, 188-195.

D. Visco Jr., R. Pophale, M. Rintoul, J.-L. Faulon, 2002, Developing a methodology for an inverse quantitative structure-activity relationship using the signature molecular descriptor, J. of Mol. Graphics and Modelling, 20, 429-438.

D. Weis, J.-L. Faulon, R. LeBorne, D. Visco Jr., 2005, The Signature Molecular Desciptor. 5. The Design of Hydrofluoroether Foam Blowing Agents Using Inverse-QSAR, Ind. Eng. Chem. Res., 44, 8883-8891. 